\begin{tabular}{|c|c|c|c|c|c|c|}
\hline \multirow{4}{*}{ Impact Factor: } & ISRA (India) & $=4.971$ & SIS (USA) & $=0.912$ & ICV (Poland) & $=6.630$ \\
\hline & ISI (Dubai, UAE & $=0.829$ & РИНЦ (Russia) & $=0.126$ & PIF (India) & $=1.940$ \\
\hline & GIF (Australia) & $=0.564$ & ESJI (KZ) & $=8.716$ & IBI (India) & $=4.260$ \\
\hline & JIF & $=1.500$ & SJIF (Morocco) & $=5.667$ & OAJI (USA) & $=0.350$ \\
\hline
\end{tabular}

\section{SOI: 1.1/TAS DOI: $10.15863 /$ TAS \\ International Scientific Journal Theoretical \& Applied Science}

p-ISSN: 2308-4944 (print) e-ISSN: 2409-0085 (online)

Year: $2020 \quad$ Issue: 03 Volume: 83

Published: $30.03 .2020 \quad$ http://T-Science.org
QR - Issue

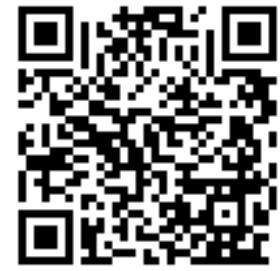

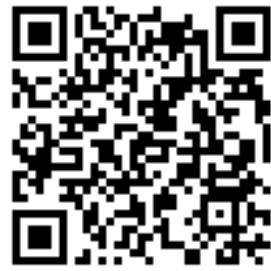

Obid Abdullayev

Samarkand State University

Docent of Department Theoretical and Applied Mechanics

Samarkand, Uzbekistan

abdullaev2006@inbox.ru

\title{
ITERATIVE METHOD FOR SOLVING MATRIX EQUATIONS FOR TWO-DIMENSIONAL PROBLEMS OF ELASTICITY THEORY
}

Abstract: An iterative method for solving matrix equations obtained by solving the problems of the linear theory of elasticity by the mixed finite element method is considered. Numerical implementation on a computer of mixed projection-grid algorithms of the FEM. It is associated with the development of effective methods for solving the corresponding discrete problems, which are represented by systems of matrix equations. Using the properties of the matrices, estimates of the rate of convergence of iterative processes are obtained.

Key words: matrix equations, mixed finite element method, projection-grid algorithms, system of matrix equations, rate of convergence.

Language: Russian

Citation: Abdullayev, O. (2020). Iterative method for solving matrix equations for two-dimensional problems of elasticity theory. ISJ Theoretical \& Applied Science, 03 (83), 149-154.

Soi: http://s-o-i.org/1.1/TAS-03-83-32 Doi: crosłef https://dx.doi.org/10.15863/TAS.2020.03.83.32

Scopus ASCC: 2600.

\section{ИТЕРАЦИОННЫЙ МЕТОД РЕШЕНИЯ МАТРИЧНЫХ УРАВНЕНИЙ ДЛЯ ДВУМЕРНЫХ ЗАДАЧ ТЕОРИИ УПРУГОСТИ}

Аннотация: Рассматривается итерационный метод решения матричных уравнений, получаемых при решении задач линейной теории упругости смешанным методом конечных элементов. Численная реализация на ЭВМ смешанных проекционно-сеточных алгоритмов МКЭ. Связано с разработкой эффективных методов решения соответствуюших дискретных задач, которые представляется системами матричных уравнений. Используя свойства матриц, получены оценки скорости сходимости итерачионных прочессов.

Ключевые слова: матричные уравнения, смешанный метод конечных элементов, проекционносеточный алгоритм, система матричных уравнений, оченка скорости сходимости.

Введение

УДК 539.3

Численная реализация на ЭВМ смешанних проекционно-сеточных алгоритмов МКЭ связано с разработкой эффективных методов решения соответствующих дискретных задач, которые представляется системами матричных линейных уравнений. При этом разработка таких методов является одним из важнейших факторов, в целом определяющих успешную реализацию смешанной МКЭ $[1,2]$.
Рассмотрим систему матричных линейных уравнений, определяющую смешанную конечно элементную аппроксимацию краевой задачи теории упругости.

$$
\left\{\begin{array}{c}
{\left[\mathrm{M}_{h}\right]\left\{\hat{\varepsilon}_{h}\right\}=\left[\mathrm{H}_{h}\right]\left\{\hat{\mathrm{u}}_{h}\right\}} \\
\left\{\hat{\sigma}_{h}\right\}=\left[\hat{\mathrm{D}}_{h}\right]\left(\left\{\hat{\varepsilon}_{h}\right\}-\left\{\hat{\zeta}_{h}\right\}\right. \\
{\left[\mathrm{H}_{h}\right]^{T}\left\{\hat{\sigma}_{h}\right\}=\left\{\hat{\rho}_{h}\right\}}
\end{array}\right.
$$

Равенства (1) - (3) могут быть записаны одним матричным уравнением относительно перемещений, т.е. 


\begin{tabular}{|c|c|c|c|c|c|c|}
\hline \multirow{4}{*}{ Impact Factor: } & ISRA (India) & $=4.971$ & SIS (USA) & $=0.912$ & ICV (Poland) & $=6.630$ \\
\hline & ISI (Dubai, UAE & $=\mathbf{0 . 8 2 9}$ & РИНЦ (Russia) & $=0.126$ & PIF (India) & $=1.940$ \\
\hline & GIF (Australia) & $=0.564$ & ESJI (KZ) & $=8.716$ & IBI (India) & $=4.260$ \\
\hline & JIF & $=1.500$ & SJIF (Morocco) & $=5.667$ & OAJI (USA) & $=0.350$ \\
\hline
\end{tabular}

$$
\left[\mathrm{A}_{h}\right]\left\{\hat{\mathrm{u}}_{h}\right\}=\left\{\hat{\mathrm{f}}_{h}\right\},
$$

где

$$
\left[\mathrm{A}_{h}\right]=\left[\mathrm{H}_{h}\right]^{T}\left[\hat{\mathrm{D}}_{h}\right]\left[\mathrm{M}_{h}\right]^{-1}\left[\mathrm{H}_{h}\right]
$$

- симметричная положительно определенная ограниченная матрица;

$$
\left\{\hat{\mathrm{f}}_{h}\right\}=\left\{\hat{\rho}_{h}\right\}+\left[\mathrm{H}_{h}\right]^{T}\left[\hat{\mathrm{D}}_{h}\right]\left\{\hat{\zeta}_{h}\right\}
$$

- вектор правой части. Для решения системы линейных алгебраических уравнений (4) могут быть использованы классические методы вычислительной алгебры, изложенные, например, в работах $[3,4]$.

Однако, использование уравнения (4) с целью нахождения решения дискретной задачи (1) - (3) является, по-видимому, нецелесообразно, т.к. в определение матрицы $\left[\mathrm{A}_{h}\right]$ входит матрица $\left[\mathrm{M}_{h}\right]^{-1}$, обратная по отношению к матрице $\left[M_{h}\right]$ . численная процедура обращения матрицы $\left[\mathrm{M}_{h}\right]$, с целью построения матрицы $\left[\mathrm{A}_{h}\right]$, является достаточно трудоемкой и неэффективной.

В настоящей работе для решения системы уравнений (1) - (3) рассматривается универсальный алгоритм, реализуемый в виде итерационной процедуры [5-7]. Для построения итерационного процесса введем в рассмотрение итерационную матрицу $\left[\mathrm{F}_{h}\right]$, обладающую следующими основными свойствами: $\left[\mathrm{F}_{h}\right]$ симметричная, положительно определенная и ограниченная матрица. Кроме того, для любого $\hat{v}_{h} \in V_{h} /\{0\}$ справедливо неравенство:

$$
\left\{\begin{array}{l}
{\left[M_{h}\right]\left\{\hat{\varepsilon}_{h}^{k+1}\right\}=\left[H_{h}\right]\left\{\hat{u}_{h}^{k}\right\}} \\
\left\{\hat{\sigma}_{h}^{k+1}\right\}=\left[\hat{D}_{h}\right]\left(\left\{\hat{\varepsilon}_{h}^{k+1}\right\}-\left\{\hat{\zeta}_{h}\right\}\right) \\
{\left[D_{h}\right]\left\{\hat{\xi}_{h}^{k+1}\right\}=\left\{\hat{\rho}_{h}\right\}-\left[H_{h}\right]^{T}\left\{\hat{\sigma}_{h}^{k+1}\right\}-\omega\left[L_{h}\right]\left\{\hat{\xi}_{h}^{k+1}\right\}} \\
\left\{\hat{u}_{h}^{k+1}\right\}=\left\{\hat{u}_{h}^{k}\right\}+\omega\left\{\hat{\xi}_{h}^{k+1}\right\}
\end{array}\right.
$$

Здесь $\omega$ - параметр, вводимый для управления сходимостью итерационного процесса.

Докажем, что при $\omega \in(0 ; 2)$ итерационный процесс (9)-(12) сходится со скоростью геометрической прогрессии независимо от выбора

$$
\begin{gathered}
\left\{\hat{v}_{h}\right\}^{T}\left[\mathrm{~A}_{h}\right]\left\{\hat{v}_{h}\right\} \leq\left\{\hat{v}_{h}\right\}^{T}\left[\mathrm{~F}_{h}\right]\left\{\hat{v}_{h}\right\}, \\
\forall \hat{v}_{h} \in V_{h} /\{0\} .
\end{gathered}
$$

Очевидно, что конкретный выбор итерационной матрицы $\left[\mathrm{F}_{h}\right]$, удовлетворяющей высшее свойствам, является классических соотношений МКЭ в форме метода перемещений. $\mathrm{B}$ таком случае, $\left[\mathrm{F}_{h}\right]$ - матрица жесткости, соответствующая МКЭ в форме метода перемещений. Однако такой выбор итерационной матрицы $\left[\mathrm{F}_{h}\right]$ не является единственно возможным.

Представим теперь итерационную матрицу $\left[\mathrm{F}_{h}\right]$ в виде разложения

$$
\left[\mathrm{F}_{h}\right]=\left[\mathrm{L}_{h}\right]+\left[\mathrm{D}_{h}\right]+\left[\mathrm{L}_{h}\right]^{T},
$$

где $\left[\mathrm{D}_{h}\right]$-диагональная (диагонально-блочная), положительно определенная, ограниченная матрица, $\left[\mathrm{L}_{h}\right]$ - строго нижняя треугольная (треугольно-блочная) матрица и $\left[\mathrm{L}_{h}\right]^{T}-$ транспонированная по отношению к $\left[\mathrm{L}_{h}\right]$ матрица. Тогда решение системы уравнений (1) (3) может быть получено с помощью следующей итерационной процедуры: начального приближения. Для этого перейдем в уравнениях (9)-(12) к векторам ошибок итерационного процесса. В результате получим [8-12]

$$
\begin{gathered}
{\left[M_{h}\right]\left(\left\{\hat{\varepsilon}_{h}^{k+1}\right\}-\left\{\hat{\varepsilon}_{h}\right\}\right)=\left[H_{h}\right]\left(\left\{\hat{u}_{h}^{k}\right\}-\left\{\hat{u}_{h}\right\}\right),} \\
\left\{\hat{\sigma}_{h}^{k+1}\right\}-\left\{\hat{\sigma}_{h}\right\}=\left[\hat{D}_{h}\right]\left(\left\{\hat{\varepsilon}_{h}^{k+1}\right\}-\left\{\hat{\varepsilon}_{h}\right\}\right),
\end{gathered}
$$

$$
\left\{\hat{u}_{h}^{k+1}\right\}-\left\{\hat{u}_{h}\right\}=\left\{\hat{u}_{h}^{k}\right\}-\left\{\hat{u}_{h}\right\}-\omega\left(\left[D_{h}\right]+\omega\left[L_{h}\right]\right)^{-1}\left[H_{h}\right]^{T}\left(\left\{\hat{\sigma}_{h}^{k+1}\right\}-\left\{\hat{\sigma}_{h}\right\}\right) .
$$

Отсюда находим 


\begin{tabular}{|c|c|c|c|c|c|c|}
\hline \multirow{4}{*}{ Impact Factor: } & ISRA (India) & $=4.971$ & SIS (USA) & $=0.912$ & ICV (Poland) & $=6.630$ \\
\hline & ISI (Dubai, UAE) & $=0.829$ & РИНЦ (Russia) & $=0.126$ & PIF (India) & $=1.940$ \\
\hline & GIF (Australia) & $=0.564$ & ESJI (KZ) & $=8.716$ & IBI (India) & $=4.260$ \\
\hline & JIF & $=1.500$ & SJIF (Morocco) & $=5.667$ & OAJI (USA) & $=0.350$ \\
\hline
\end{tabular}

$$
\left\{\hat{u}_{h}^{k+1}\right\}-\left\{\hat{u}_{h}\right\}=\left\{\hat{u}_{h}^{k}\right\}-\left\{\hat{u}_{h}\right\}-\omega\left(\left[D_{h}\right]+\omega\left[L_{h}\right]\right)^{-1}\left[A_{h}\right]^{T}\left(\left\{\hat{\sigma}_{h}^{k+1}\right\}-\left\{\hat{\sigma}_{h}\right\}\right) .
$$

Тогда матрица перехода $\left[T_{h}(\omega)\right]$, соответствующая итерационному процессу (9)(12), определяется выражением:

$$
\left[T_{h}(\omega)\right]=\left[\mathrm{E}_{h}\right]-\omega\left(\left[D_{h}\right]+\omega\left[L_{h}\right]\right)^{-1}\left[A_{h}\right] .
$$

Здесь и ниже $\left[\mathrm{E}_{h}\right]$ - единичная матрица в $\left[V_{h}\right]$ следовательно, уравнение для последовательности векторов ошибок $\left\{\hat{u}_{h}^{k}\right\}-\left\{\hat{u}_{h}\right\}$ можно представить в следующем виде

$$
\left\{\hat{u}_{h}^{k}\right\}-\left\{\hat{u}_{h}\right\}=\left[T_{h}^{k}(\omega)\right] \cdot\left(\left\{\hat{u}_{h}^{o}\right\}-\left\{\hat{u}_{h}\right\}\right),
$$

где $\left\{\hat{u}_{h}^{o}\right\}$ - вектор, соответствующий начальному приближению. Из (18) получаем:

$\left\|\left\{\hat{u}_{h}^{k}\right\}-\left\{\hat{u}_{h}\right\}\right\| \leq\left\|\left[T_{h}(\omega)\right]^{k}\right\| \cdot\left\|\left\{\hat{u}_{h}^{o}\right\}-\left\{\hat{u}_{h}\right\}\right\|$,

где $\|\cdot\|$ - некоторая норма. Кроме того, учитывая, что справедливо неравенство

$$
\left\|\left[T_{h}(\omega)\right]^{k}\right\| \leq\left\|\left[T_{h}(\omega)\right]\right\|^{k}
$$

приходим к оценке:

$\left\|\left\{\hat{u}_{h}^{k}\right\}-\left\{\hat{u}_{h}\right\}\right\| \leq\left\|\left[T_{h}(\omega)\right]\right\|^{k} \cdot\left\|\left\{\hat{u}_{h}^{o}\right\}-\left\{\hat{u}_{h}\right\}\right\|$,

следовательно, условие

$$
\begin{gathered}
\left\|\left[T_{h}(\omega)\right]\right\|<1 \quad \text { (22) } \begin{array}{c}
{\left[T_{h}(\omega)\right] . \text { Тогда в соответствии с определением }} \\
\text { матрицы }\left[T_{h}(\omega)\right] \text { и }\left[A_{h}\right] \text { - нормы имеем: }
\end{array} \\
\left\|\left[T_{h}(\omega)\right]\right\|_{\left[A_{h}\right]}=\sup _{\left\{\hat{\vartheta}_{h}\right\} \neq\{0\}} \frac{\left\{\hat{\vartheta}_{h}\right\}^{T}\left[T_{h}(\omega)\right]^{T}\left[A_{h}\right]\left[T_{h}(\omega)\right]\left\{\hat{\vartheta}_{h}\right\}}{\left\{\hat{\vartheta}_{h}\right\}^{T}\left[A_{h}\right]\left\{\hat{\vartheta}_{h}\right\}}=\sup _{\left\{\hat{w}_{h}\right\} \neq\{0\}} \frac{\left\{\hat{w}_{h}\right\}^{T}\left[\breve{T}_{h}(\omega)\right]^{T}\left[\breve{T}_{h}(\omega)\right]\left\{\hat{w}_{h}\right\}}{\left\{\hat{w}_{h}\right\}^{T}\left\{\hat{w}_{h}\right\}}, \quad \text { (27) }
\end{gathered}
$$

необходимо и достаточно для сходимости итерационного процесса. Оценим теперь $\left\|\left[T_{h}(\omega)\right]\right\|$. Для этого учтем, что для любого вектора $\hat{\vartheta}_{h} \in V_{h} \backslash\{0\}$ имеет место неравенство:

$$
\left\{\hat{\vartheta}_{h}\right\}^{T}\left[A_{h}\right]\left\{\hat{\vartheta}_{h}\right\} \geq \stackrel{o}{a} \min _{\operatorname{mo}}\left\{\hat{\vartheta}_{h}\right\}^{T}\left[D_{h}\right]\left\{\hat{\vartheta}_{h}\right\}
$$

Очевидно, что $a_{\min }$ есть нижняя граница спектра симметричной положительно определенной ограниченной матрицы

$$
\left[D_{h}\right]^{-1 / 2}\left[A_{h}\right]\left[D_{h}\right]^{1 / 2} .
$$

И, следовательно, константа $a_{\text {min }}$ всегда существует. Равенство

$\left\|\left\{\hat{\vartheta}_{h}\right\}\right\|_{\left[A_{h}\right]}=\left(\left\{\hat{\vartheta}_{h}\right\}^{T}\left[A_{h}\right]\left\{\hat{\vartheta}_{h}\right\}\right)^{1 / 2} ; \forall \hat{\vartheta}_{h} \in V_{h}$.

определяет энергетическую норму в пространстве векторов ошибок $\left\{\hat{\vartheta}_{h}^{k}\right\}-\left\{\hat{\vartheta}_{h}\right\}$

Соответственно соотношение

$$
\left\|\left[T_{h}(\omega)\right]\right\|_{\left[A_{h}\right]}=\sup _{\left\{\hat{\vartheta}_{h}\right\} \neq 0} \frac{\left\|\left[T_{h}(\omega)\right]\left\{\hat{\vartheta}_{h}\right\}\right\|_{\left[A_{h}\right]}}{\|\{\hat{\vartheta}\}\|_{\left[A_{h}\right]}} .
$$

определяет $\left[A_{h}\right]-$ норму матрицы перехода

здесь

$$
\left[\breve{T}_{h}(\omega)\right]=\left[A_{h}\right]^{1 / 2}\left[T_{h}(\omega)\right]\left[A_{h}\right]^{-1 / 2}=\left[\mathrm{E}_{h}\right]-\omega\left[A_{h}\right]^{1 / 2}\left(\left[D_{h}\right]+\omega\left[L_{h}\right]\right)^{-1}\left[A_{h}\right]^{1 / 2}
$$

и $\left\{\hat{w}_{h}\right\}=\left[A_{h}\right]^{1 / 2}\left\{\hat{\vartheta}_{h}\right\}$.

$$
\text { Кроме того, }\left[A_{h}\right]^{1 / 2} \text { и } \quad\left[A_{h}\right]^{-1 / 2} \text { - }
$$

положительно определенные ограниченные матрицы, определяемые соотношениями:

$$
\left[A_{h}\right]^{1 / 2} \cdot\left[A_{h}\right]^{1 / 2}=\left[A_{h}\right],\left[A_{h}\right]^{-1 / 2} \cdot\left[A_{h}\right]^{-1 / 2}=\left[A_{h}\right]^{-1} .
$$

Следовательно, линейное преобразование (29) невырожденное, т.е. $\left\{\hat{w}_{h}\right\} \neq\{0\}$.
Введем в рассмотрение треугольные положительно-определенные и ограниченные матрицы $\left[C_{h}\right]$ и $\left[C_{h}\right]^{T}$ с помощью выражений: 


\begin{tabular}{|c|c|c|c|c|c|c|}
\hline \multirow{4}{*}{ Impact Factor: } & ISRA (India) & $=4.971$ & SIS (USA) & $=0.912$ & ICV (Poland) & $=6.630$ \\
\hline & ISI (Dubai, UAE & $=0.829$ & РИНЦ (Russia) & $=0.126$ & PIF (India) & $=1.940$ \\
\hline & GIF (Australia) & $=0.564$ & ESJI (KZ) & $=8.716$ & IBI (India) & $=4.260$ \\
\hline & JIF & $=1.500$ & SJIF (Morocco) & $=5.667$ & OAJI (USA) & $=0.350$ \\
\hline
\end{tabular}

$$
\begin{aligned}
& {\left[C_{h}\right]=\frac{1}{2}\left[D_{h}\right]+\left[L_{h}\right],} \\
& {\left[C_{h}\right]^{T}=\frac{1}{2}\left[D_{h}\right]+\left[L_{h}\right]^{T}\left[F_{h}\right]=\left[C_{h}\right]+\left[C_{h}\right]^{T} .} \\
& {\left[\breve{T}_{h}(\omega)\right]=\left[E_{h}\right]-\alpha\left[A_{h}\right]^{1 / 2}\left(\left[D_{h}\right]+\alpha\left[C_{h}\right]\right)^{-1}\left[A_{h}\right]^{1 / 2} .} \\
& \alpha=\frac{2 \omega}{2-\omega} ; \alpha \in(0 ; \infty), \forall \omega \in(0 ; 2) . \\
& \left\{\hat{w}_{h}\right\}^{T}\left[\breve{T}_{h}(\omega)\right]^{T}\left[\breve{T}_{h}(\omega)\right]\left\{\hat{w}_{h}\right\} \leq\left\{\hat{w}_{h}\right\}^{T}\left\{\hat{w}_{h}\right\}-2 \alpha\left\{\hat{z}_{h}\right\}^{T}\left[D_{h}\right]\left\{\hat{z}_{h}\right\} . \\
& \left\{\hat{z}_{h}\right\}=\left(\left[D_{h}\right]+\alpha\left[C_{h}\right]\right)^{-1}\left[A_{h}\right]^{1 / 2}\left\{\hat{w}_{h}\right\} . \\
& \left\{\hat{w}_{h}\right\}^{T}\left[\breve{T}_{h}(\omega)\right]^{T}\left[\breve{T}_{h}(\omega)\right]\left\{\hat{w}_{h}\right\} \leq\left\{\hat{w}_{h}\right\}^{T}\left\{\hat{w}_{h}\right\}-2 \alpha\left\{\hat{w}_{h}\right\}^{T}\left[\Pi_{h}(\alpha)\right]\left\{\hat{w}_{h}\right\} . \\
& \text { Здесь }\left[\Pi_{h}(\alpha)\right] \text { - симметричная матрица, } \\
& \text { определяемая выражением: } \\
& {\left[\Pi_{h}(\alpha)\right]=\left[A_{h}\right]^{1 / 2}\left(\left[D_{h}\right]+\alpha\left[C_{h}\right]^{T}\right)^{-1}\left[D_{h}\right]\left(\left[D_{h}\right]+\alpha\left[C_{h}\right]\right)^{-1}\left[A_{h}\right]^{1 / 2} .}
\end{aligned}
$$

Таким образом, согласно равенствам (27) и неравенству (36) приходим к оценке:

$$
\left\|\left[T_{h}(\omega)\right]\right\|_{\left[A_{h]}\right.} \leq 1-2 \alpha \inf _{\left\{\hat{w}_{h}\right\} \neq\{0\}} \frac{\left\{\hat{w}_{h}\right\}^{T}\left[\Pi_{h}(\alpha)\right]\left\{\hat{w}_{h}\right\}}{\left\{\hat{w}_{h}\right\}^{T}\left\{\hat{w}_{h}\right\}} .
$$

Для оценки второго слагаемого этого неравенства рассмотрим спектральную задачу

$$
\left[\Pi_{h}(\alpha)\right]\left\{\hat{y}_{h s}\right\}=\lambda_{h s}\left(\left[\Pi_{h}(\alpha)\right]\right)\left\{\hat{y}_{h s}\right\} .
$$

Поскольку матрица $\left[\Pi_{h}(\alpha)\right]$ симметричная, значения $\quad \lambda_{h s}\left(\left[\Pi_{h}(\alpha)\right]\right)$ вещественны. $\mathrm{B}$ соответствии с определением матриц $\left[\Pi_{h}(\alpha)\right]$ спектральные задачи (39) преобразуется к следующему виду

$$
\left[A_{h}\right]\left\{\hat{z}_{h s}\right\}=\lambda_{h s}\left(\left[\Pi_{h}(\alpha)\right]\right)\left(\left[D_{h}\right]+\alpha\left[F_{h}\right]+(\alpha)^{2}\left[C_{h}\right]\left[D_{h}\right]^{-1}\left[C_{h}\right]^{T}\right)\left\{\hat{z}_{h s}\right\},
$$

где

$$
\left\{\hat{z}_{h s}\right\}=\left[A_{h}\right]^{-\frac{1}{2}}\left\{\hat{y}_{h s}\right\}
$$

Из тождества (40) находим $\lambda_{h s}\left(\left[\Pi_{h}(\alpha)\right]\right)$ и оценим его снизу. Так как для любого $\hat{z}_{h s} \in V_{h} \backslash\{0\}$ справедливо неравенство:

$$
\left\{\hat{z}_{h s}\right\}^{T}\left[C_{h}\right]\left[D_{h}\right]^{-1}\left[C_{h}\right]^{T}\left\{\hat{z}_{h s}\right\} \leq \frac{0}{4}\left\{\hat{z}_{h s}\right\}^{T}\left[A_{h}\right]\left\{\hat{z}_{h s}\right\}
$$

Очевидно, что $\frac{0}{4}$ - есть верхняя граница спектра симметричной, положительно определенной, ограниченной матрицы:
$\left[A_{h}\right]^{-\frac{1}{2}}\left[C_{h}\right]\left[D_{h}\right]^{-1}\left[C_{h}\right]^{T}\left[A_{h}\right]^{-\frac{1}{2}} \quad$ (43)
0
и следовательно, константа $\frac{\Delta}{4}$ всегда существует.

Используя неравенство (42) имеем: 


\begin{tabular}{|c|c|c|c|c|c|c|}
\hline \multirow{4}{*}{ Impact Factor: } & ISRA (India) & $=4.971$ & SIS (USA) & $=0.912$ & ICV (Poland) & $=6.630$ \\
\hline & ISI (Dubai, UAE) & $=0.829$ & РИНЦ (Russia) & $=0.126$ & PIF (India) & $=1.940$ \\
\hline & GIF (Australia) & $=0.564$ & ESJI (KZ) & $=8.716$ & IBI (India) & $=4.260$ \\
\hline & JIF & $=1.500$ & SJIF (Morocco) & $=5.667$ & OAJI (USA) & $=0.350$ \\
\hline
\end{tabular}

$$
\lambda_{h s}\left(\left[\Pi_{h}(\alpha)\right]\right) \geq \frac{\left\{\hat{z}_{h s}\right\}^{T}\left[A_{h}\right]\left\{\hat{z}_{h s}\right\}}{\left\{\hat{z}_{h s}\right\}^{T}\left[D_{h}\right]\left\{\hat{z}_{h s}\right\}+\alpha\left\{\hat{z}_{h s}\right\}^{T}\left[F_{h}\right]\left\{\hat{z}_{h s}\right\}+\frac{\alpha^{2} \Delta^{0}}{4}\left\{\hat{z}_{h s}\right\}^{T}\left[A_{h}\right]\left\{\hat{z}_{h s}\right\}}
$$

Используя неравенство:

$$
\left\{\hat{z}_{h s}\right\}^{T}\left[A_{h}\right]\left\{\hat{z}_{h s}\right\} \geq \Delta_{\min }\left\{\hat{z}_{h s}\right\}^{T}\left[F_{h}\right]\left\{\hat{z}_{h s}\right\} .
$$

(44) приведем к виду:

$$
\lambda_{h s}\left(\left[\Pi_{h}(\alpha)\right]\right) \geq \frac{\inf _{\left\{\hat{x}_{h s}\right\} \neq\{0\}}\left\{\hat{x}_{h s}\right\}^{T}\left[A_{h}\right]\left\{\hat{x}_{h s}\right\}}{1+\alpha\left(\frac{1}{\Delta_{\min }}+\frac{\alpha \Delta}{4}\right) \inf _{\left\{\hat{x}_{h s}\right\} \neq\{0\}}\left\{\hat{x}_{h s}\right\}^{T}\left[A_{h}\right]\left\{\hat{x}_{h s}\right\}} .
$$

Используя теперь тот факт, что функция $\frac{t}{1+b t}$ монотонно возрастает при $t \in(0 ;+\infty)$ для любой положительной константы $b$ получим оценку

$$
\lambda_{h s}\left(\left[\Pi_{h}(\alpha)\right]\right) \geq \frac{a_{\min }}{1+\alpha\left(\frac{1}{\Delta_{\min }}+\frac{\alpha \Delta}{4}\right) a_{\min }}
$$

Таким образом, согласно неравенством (38) и (47) имеем:

$$
\left\|\left[T_{h}(\omega)\right]\right\|_{\left[A_{h}\right]}^{2} \leq 1-\frac{2 \alpha a_{\min }}{1+\alpha\left(\frac{1}{\Delta_{\min }}+\frac{\alpha \Delta}{4}\right){ }_{a_{\min }}^{0}} .
$$

отсюда находим:

$$
\left[\left(\left\{\hat{u}_{h}^{k}\right\}-\left\{\hat{\mathbf{u}}_{h}\right\}\right)^{T}\left(\left\{\hat{u}_{h}^{k}\right\}-\left\{\hat{\mathbf{u}}_{h}\right\}\right)\right]^{1 / 2} \leq \sqrt{\mu\left(\left[\mathrm{A}_{h}\right]\right)} q^{k}(\alpha)\left[\left(\left\{\hat{u}_{h}^{0}\right\}-\left\{\hat{\mathbf{u}}_{h}\right\}\right)^{T}\left(\left\{\hat{u}_{h}^{0}\right\}-\left\{\hat{\mathbf{u}}_{h}\right\}\right)\right]^{1 / 2} .
$$

Более того, используя (52) и на основании неравенств приведенного в $[10 ; 11 ; 12]$ получаем оценку скорости сходимости итерационного

$$
\left\|\left[T_{h}(\omega)\right]\right\|_{\left[A_{h}\right]} \leq q(\alpha)<1 .
$$

Тогда в соответствии с неравенствами (21), (48) получаем оценку скорости сходимости итерационного процесса:

$\left\|\left\{\hat{u}_{h}^{k}\right\}-\left\{\hat{\mathbf{u}}_{h}\right\}\right\|_{\left[\mathrm{A}_{h}\right]} \leq q^{k}(\alpha) \cdot\left\|\left\{\hat{u}_{h}^{0}\right\}-\left\{\hat{\mathbf{u}}_{h}\right\}\right\|_{\left[\mathrm{A}_{h}\right]}$.

Учитывая теперь, что $\left[\mathrm{A}_{h}\right]$ - симметричная, положительно определенная и ограниченная матрица, причем существуют два вещественных положительных чисел $a_{\min }(h), a_{\max }(h)$ такие, что для любого $\hat{\vartheta}_{h} \in V_{h} \backslash\{0\}$ справедливы неравенства $a_{\text {min }}(h)\left\{\hat{v}_{h}\right\}^{T}\left\{\hat{v}_{h}\right\} \leq\left\|\left\{\hat{v}_{h}\right\}\right\|_{\left[\mathrm{A}_{h}\right]}^{2} \leq a_{\max }(h)\left\{\hat{v}_{h}\right\}^{T}\left\{\hat{v}_{h}\right\}$

Согласно неравенствам (50), (51) имеем оценку скорости сходимости итерационного процесса относительно векторов ошибок перемещений:

$$
\left\|u_{h}^{k}-u_{h}\right\|_{\left[L_{2}(\Omega)\right]^{n}} \leq \frac{c_{0} c_{1} c_{3}}{d_{0} c_{2} h_{\min } \frac{h_{\min }{\sqrt[n]{\Delta_{0}}}^{k}\left\|u_{h}^{0}-u_{h}\right\|_{\left[L_{2}(\Omega)\right]^{n}}}{n}} .
$$

Аналогичные оценки могут быть получены и для ошибок $\sigma_{h}^{k}-\sigma_{h}$ и $\varepsilon_{h}^{k}-\varepsilon_{h}$ в метрике пространства $\mathrm{X}$. процесса относительно ошибок $u_{h}^{k}-u_{h}$ в метрике пространства $\left[\mathrm{L}_{2}(\Omega)\right]^{n}$, т.е.

$$
\left[\left(\left\{\hat{\sigma}_{h}^{k}\right\}-\left\{\hat{\sigma}_{h}\right\}\right)^{T}\left[\mathrm{M}_{h}\right]\left(\left\{\hat{\varepsilon}_{h}^{k}\right\}-\left\{\hat{\varepsilon}_{h}\right\}\right)\right]^{1 / 2} \leq q^{k}(\alpha)\left[\left(\left\{\hat{\sigma}_{h}^{0}\right\}-\left\{\hat{\sigma}_{h}\right\}\right)^{T}\left[\mathrm{M}_{h}\right]\left(\left\{\hat{\varepsilon}_{h}^{0}\right\}-\left\{\hat{\varepsilon}_{h}\right\}\right)\right]^{1 / 2} .
$$




\begin{tabular}{|c|c|c|c|c|c|c|}
\hline \multirow{4}{*}{ Impact Factor: } & ISRA (India) & $=4.971$ & SIS (USA) & $=0.912$ & ICV (Poland) & $=6.630$ \\
\hline & ISI (Dubai, UAE & $=0.829$ & РИНЦ (Russia & $=0.126$ & PIF (India) & $=1.940$ \\
\hline & GIF (Australia) & $=0.564$ & ESJI (KZ) & $=8.716$ & IBI (India) & $=4.260$ \\
\hline & JIF & $=1.500$ & SJIF (Morocec & $=5.667$ & OAJI (USA) & $=0.350$ \\
\hline
\end{tabular}

Используя уравнение (14) и свойств матриц

$\left[\mathrm{M}_{h}\right]$ и $\left[\hat{\mathrm{D}}_{h}\right]$ получаем оценки скорости

сходимости итерационного процесса относительно векторов ошибок напряжений и деформаций:

$$
\begin{gathered}
{\left[\left(\left\{\hat{\sigma}_{h}^{k}\right\}-\left\{\hat{\sigma}_{h}\right\}\right)^{T}\left[M_{h}\right]\left(\left\{\hat{\sigma}_{h}^{k}\right\}-\left\{\hat{\sigma}_{h}\right\}\right)\right]^{\frac{1}{2}} \leq \sqrt{\frac{\Delta_{0}}{\delta_{0}}} q^{k}(\alpha)\left[\left(\left\{\hat{\sigma}_{h}^{0}\right\}-\left\{\hat{\sigma}_{h}\right\}\right)^{T}\left[M_{h}\right]\left(\left\{\hat{\sigma}_{h}^{0}\right\}-\left\{\hat{\sigma}_{h}\right\}\right)\right]^{\frac{1}{2}}} \\
{\left[\left(\left\{\hat{\varepsilon}_{h}^{k}\right\}-\left\{\hat{\varepsilon}_{h}\right\}\right)^{T}\left[M_{h}\right]\left(\left\{\hat{\varepsilon}_{h}^{k}\right\}-\left\{\hat{\varepsilon}_{h}\right\}\right)\right]^{\frac{1}{2}} \leq \sqrt{\frac{\Delta_{0}}{\delta_{0}}} q^{k}(\alpha)\left[\left(\left\{\hat{\varepsilon}_{h}^{0}\right\}-\left\{\hat{\varepsilon}_{h}\right\}\right)^{T}\left[M_{h}\right]\left(\left\{\hat{\varepsilon}_{h}^{0}\right\}-\left\{\hat{\varepsilon}_{h}\right\}\right)\right]^{\frac{1}{2}}}
\end{gathered}
$$

Тогда согласно определению нормы в пространствах $\mathrm{Z}_{h}$ и $\mathrm{X}$ имеем:

$$
\begin{aligned}
& \left\|\sigma_{h}^{k}-\sigma_{h}\right\|_{X} \leq \sqrt{\frac{\Delta_{0}}{\delta_{0}}} q^{k}(\omega) \cdot\left\|\sigma_{h}^{0}-\sigma_{h}\right\|_{X} ; \\
& \left\|\varepsilon_{h}^{k}-\varepsilon_{h}\right\|_{X} \leq \sqrt{\frac{\Delta_{0}}{\delta_{0}}} q^{k}(\omega) \cdot\left\|\varepsilon_{h}^{0}-\varepsilon_{h}\right\|_{X} .
\end{aligned}
$$

Таким образом, справедлива следующая теорема.
Tеорема. Если итерационная матрица $\left[\mathrm{F}_{h}\right]$ удовлетворяет перечисленным выше условиям, $\left[\mathrm{A}_{h}\right]$ - симметричная, положительно определенная, ограниченная матрица и $\omega \in(0 ; 2)$ , итерационный процесс (9)-(12) сходится со скоростью геометрической прогрессии независимо от выбора начального приближения. При этом имеет место оценки (53), (57), (58).

\section{References:}

1. Amosov, A.A., Dubinskiy, Yu.A., \& Kopchenova, N.V. (2014). Vichislitelniye metodi. Izd. Lan, p.672.

2. Baxvalov, I.S., Jidkov, N.P., \& Kobelkov, G.M. (2011). Chislenniye metodi. (p.640). M.: Izd-vo BINOM. Laboratoriya znaniy.

3. Verbjiskiy, V.M. (2009). Vichislitelnaya lineynaya algebra. (p.315). Moscow: Visshaya shkola.

4. Demidovich, B.P., \& Maron, I. A. (2009). Osnovi vichislitelnoy matematiki. (p.672). SPb.: Lan.

5. Djordj, A., L. Dj. (1984). Chislennoye resheniye bolshix razrejennix sistem uravneniy. (p.333). Moscow: Mir.

6. Kalitkin, N.N. (2011). Chislenniye metodi. (p.592). SPb.: BXV. - Peterburg.

7. Kireyev, V.I. (2008). Chislenniye metodi v primerax i zadachax. Panteleyev. (p.408). Moscow: Visshaya shkola.
8. Marchuk, G.I., \& Agoshkov, V.I. (1981). Vvedeniye $v$ proyeksionno-setochniye metodi. (p.416). Moscow: Nauka.

9. Morozkin, N.D., Abdullayev, O., Nugumanov, E.R., Axmetshina, G.A., \& Kolonskix, D.M. (2008). Smeshannaya proyeksionno-setochnaya sxema dlya resheniya zadach teorii uprugosti. Vestnik BashGU, №1, pp.4-8.

10. Saad, Y. (2003). Iterative Methods for Spores Linear System. (p.546). STAN.

11. Uotkins, D.S. (2006). Osnovi matrichnix vichisleniy. (p.672). Moscow: BINOM. Laboratoriya znaniy.

12. Chirkov, A.Yu. (2005). Primeneniye v konechno-elementnix raschetax modifisirovannogo algoritma metoda sopryajennix gradiyentov. Problem prochnosti, №6, Kiyev, pp. 89-102. 